\title{
HARPing on about the DNA damage response during replication
}

\author{
Robert Driscoll and Karlene A. Cimprich ${ }^{1}$ \\ Department of Chemical and Systems Biology, Stanford University, Stanford, California 94305, USA
}

\begin{abstract}
In this issue of Genes \& Development, four papers report that the annealing helicase HepA-related protein (HARP, also known as SMARCAL1 [SWI/SNF-related, matrixassociated, actin-dependent regulator of chromatin, subfamily a-like 1]) binds directly to the ssDNA-binding protein Replication protein A (RPA) and is recruited to sites of replicative stress. Knockdown of HARP results in hypersensitivity to multiple DNA-damaging agents and defects in fork stability or restart. These exciting insights reveal a key new player in the S-phase DNA damage response.
\end{abstract}

All cells have evolved elaborate, coordinated responses to ensure genome integrity. Failure to accurately protect against or repair lesions that occur from exogenous or endogenous insults results in the accumulation of mutations and genomic instability. Replication is a uniquely susceptible time for the cell, as the replisome can stall at DNA lesions or certain chromosomal loci (Lambert and Carr 2005; Tourriere and Pasero 2007). In such cases, cells must protect the stability of the stalled fork in order to assist and effect fork restart. Fork instability can lead to dissociation of the polymerase and other replisome components in a process termed fork collapse (Branzei and Foiani 2009). This can lead to exposure of ssDNA and inappropriate processing and breakage, events that are increasingly linked to aberrant recombination and chromosomal translocations (Kolodner et al. 2002). Accordingly, many human genetic syndromes that lead to cancer predisposition are caused by mutations in genes whose products are central to protecting genome integrity during S phase (Hickson 2003; Kastan and Bartek 2004).

ssDNA accumulates at stably stalled replication forks (Sogo et al. 2002) and is necessary for activation of ATR (ATM [ataxia-telangiectasia mutated] and Rad3-related), a checkpoint kinase that directs the DNA damage response (Cimprich and Cortez 2008). ssDNA is also needed to recruit many repair proteins to the site of damage, and it is an intermediate in many types of DNA repair.

[Keywords: SMARCAL1; HARP; DNA damage response; RPA; annealing helicase; DNA replication]

${ }^{1}$ Corresponding author.

E-MAIL cimprich@stanford.edu; FAX (650) 725-4665.

Article is online at http://www.genesdev.org/cgi/doi/10.1101/gad.1860609.
Many recent studies have highlighted the mechanism by which ssDNA is generated at stalled forks and at doublestrand breaks (DSBs), but how its generation is kept in check and the consequences of its deregulation are not known. In this issue of Genes \& Development, four groups (Bansbach et al. 2009; Ciccia et al. 2009; Yuan et al. 2009; Yusufzai et al. 2009) link a previously identified annealing helicase, HARP (HepA-related protein)/ SMARCAL1 (SWI/SNF-related, matrix-associated, actindependent regulator of chromatin, subfamily a-like 1) to the replication fork through an interaction with Replication protein A (RPA)-coated ssDNA. Their work suggests that HARP protects stalled forks by minimizing the accumulation of ssDNA, and it illuminates how HARP is recruited to the fork, as well as the consequences of its action on fork stability, fork progression, and recovery from replication fork arrest.

\section{Checkpoint activation during $S$ phase}

In response to defects in fork progression or after forks encounter sites of DNA damage, cells activate signal transduction pathways known as checkpoints. The S-phase checkpoints must sense stalled replication forks and many different types of DNA damage during $S$ phase in order to direct appropriate responses (Branzei and Foiani 2007). A majority of data indicate that the checkpoint-activating structure contains ssDNA coated with the ssDNA-binding complex RPA, a heterotrimeric complex composed of Rpa1, Rpa2, and Rpa3 (Zou and Elledge 2003; Cimprich and Cortez 2008). RPA plays a central role in DNA metabolic pathways that involve a ssDNA intermediate, such as recombination, DNA replication, and DNA repair (Zou et al. 2006). Although RPA-coated ssDNA is present at normal replication forks, the amount of ssDNA increases at stalled forks due to a dissociation of the activities of the replicative MCM helicase and DNA polymerases (Pacek and Walter 2004). DNA processing during repair also generates ssDNA; homologous recombination repair of DNA DSBs is initiated by $5^{\prime}$-to- $3^{\prime}$ degradation of the broken DNA ends to create 3 ' ssDNA tails (Paques and Haber 1999).

RPA-coated ssDNA recruits a key regulatory protein in the replication checkpoint, ATR, to stalled or broken forks through a direct interaction with its binding partner, ATR-interacting protein (ATRIP) (Zou and Elledge 2003). 
However, RPA-coated ssDNA is not sufficient for ATR kinase activation (Zou 2007). As a result of the uncoupling of the polymerase and helicase activities, stalled polymerases also generate a primer next to this stretch of RPAcoated ssDNA, on which the Rad9-Rad1-Hus1 (9-1-1) complex, a heterotrimeric ring-shaped molecule, is loaded by a damage-specific clamp loader (Majka and Burgers 2004). In the presence of RPA, this is thought to occur on the 5' -primer end (Ellison and Stillman 2003; Majka et al. 2006). Loading of the 9-1-1 complex is linked to ATR activation by the critical intermediary protein TopBP1, which binds the phosphorylated C-terminal tail of Rad9 and directly binds both ATRIP and ATR, resulting in ATR activation (Kumagai et al. 2006; Delacroix et al. 2007; Mordes et al. 2008). After assembly and subsequent activation at a damaged or stalled replication fork, ATR coordinates the cellular response to this fork through phosphorylation of a large number of target substrates. The best-studied substrate, the effector kinase Chk1, functions to signal DNA damage to the cell through numerous targets, and together these kinases block cell cycle progression, down-regulate origin firing, stabilize stalled forks, and facilitate replication resumption or the repair of collapsed forks (Branzei and Foiani 2005; Cimprich and Cortez 2008).

\section{Stabilizing the stalled fork}

It is currently unclear exactly how stabilization of the fork is achieved, but in the absence of ATR, stalled replication forks are unstable and can collapse into a variety of deleterious structures that can prevent replication resumption and lead to unscheduled recombination (Paulsen and Cimprich 2007). Although the ATR pathway may act in several ways to stabilize forks, one critical role for ATR is to promote the stable association of DNA polymerases with stalled forks, presumably preventing the exposure of nascent strands and their rearrangement into regressed forks (Branzei and Foiani 2005; Paulsen and Cimprich 2007). These structures might be targeted by nucleases to form DSBs, and although these nucleases may be important for the repair of collapsed replication forks, excessive break formation at the fork may lead to illegitimate recombination and genomic instability (Kolodner et al. 2002; Admire et al. 2006). The precise targets for ATR's kinase activity at the fork are largely unknown, but likely substrates include replisome components such as RPA and subunits of the MCM helicase (Cortez et al. 2004; Olson et al. 2006). Regulation of the MCM helicase after fork stalling is an attractive mechanism to prevent excessive unwinding and ssDNA formation, which could make the fork more susceptible to damage. Whether this is achieved through direct phosphorylation of the MCM subunits or through the action of other enzymes is not known.

Checkpoint-independent mechanisms also contribute to fork stability. For example, in yeast, the replisomeassociated Mrc1/Tof1/Csm3 complex is believed to play a structural role in fork maintenance by helping to coordinate MCM helicase and polymerase activities and thereby prevent excessive unwinding of the MCM helicase during replication (Katou et al. 2003). Similar events are mediated by an analogous complex (Tim/Tipin) in mammalian cells (Smith et al. 2009). The RecQ helicase Sgs 1 has been reported to maintain association of DNA polymerases at stalled forks (Cobb et al. 2003), and the highly conserved Mre11-Rad50-Xrs2 complex has been shown to be critical for replisome stability, possibly via a role in promoting sister chromatin cohesion (TittelElmer et al. 2009). Chromatin structure at the fork also plays a role in fork stabilization. For example, in yeast, deletion of histone acetyltransferase Rtt109 or histone chaperone Asf1 results in a loss of fork integrity due to a loss of histone H3 Lys56 acetylation (Driscoll et al. 2007; Han et al. 2007a,b), a mark present on newly synthesized histones (Masumoto et al. 2005).

Replication forks encountering damage-induced replication blocks may have several choices for restart or resumption of DNA replication, depending on the nature of the lesion and the DNA structures formed at the fork as the result of the collision with the lesion (Heller and Marians 2006). In many cases, replication can go to completion through the action of neighboring forks that converge on the stalled fork. Alternatively, post-replication repair or DNA damage tolerance mechanisms can facilitate the completion of replication (Barbour and Xiao 2003; Smirnova and Klein 2003; Chang and Cimprich 2009). In these processes, replication is promoted by direct bypass of the lesion using either specialized polymerases or damage-bypass mechanisms, or by restarting replication downstream from the lesion. Although the latter leaves behind single-stranded gaps, these gaps can be filled by translesion synthesis polymerases, or by template switch mechanisms that use the newly synthesized DNA strand on the sister chromatid as a template for DNA synthesis across the gap. Finally, recombinationbased mechanisms can assist in completion of replication if the stalled replication fork is processed to a DSB (McGlynn and Lloyd 2002).

\section{HARP, an ATP-dependent annealing helicase}

The ubiquitous Snf2 family of ATPases acts in variety of DNA metabolic processes and is comprised of proteins with a DExx-box helicase-related motif similar to that found in Saccharomyces cerevisiae Snf2 (Flaus et al. 2006). Although most family members do not appear to act as helicases, some family members display atypical helicase activity while others are capable of ATP-dependent DNA translocation, an activity that may generate DNA distortion or disrupt chromatin as well as other DNA-protein interactions (Durr et al. 2006). Most family members also contain one or more domains other than the helicase domain, a number of which are involved in the recognition of nucleosome post-translational modifications. Some Snf2 family members are intimately involved in DNA replication and the repair of various DNA lesions and DSBs. For example, Ino80, part of a large chromatin remodelling complex, has been shown recently to be required for efficient replication fork progression, 
replication fork stability, and fork recovery from checkpoint-induced arrest (Papamichos-Chronakis and Peterson 2008; Shimada et al. 2008). Interestingly, the E3 ubiquitin ligase and Snf2 family member Rad5 is required for postreplication repair, and has a DNA helicase activity that is specialized for replication fork regression (Blastyak et al. 2007).

HARP, also known as SMARCAL1 and DNA-dependent ATPase A, is a distant member of the Snf2 family that has been shown recently to have the unique ability to catalyze the ATP-dependent reannealing of ssDNA bubbles coated with RPA (Yusufzai and Kadonaga 2008). It has also been demonstrated that the ATPase activity of HARP is activated by DNA structures with dsDNAssDNA junctions (Hockensmith et al. 1986; Muthuswami et al. 2000), including model replication fork structures (Yusufzai and Kadonaga 2008). While these findings raise the possibility that HARP acts at replication forks to reanneal ssDNA, such structures are also present during DNA repair and transcription, and, until now, where and when in the cell HARP might act was unknown. In many ways, more was known about the physiological importance of HARP since it was discovered that mutations in this gene, including some that compromise the ATPase activity of the protein, result in the autosomal recessive disorder Schimke immunoosseous dysplasia (SIOD) (Boerkoel et al. 2000). SIOD patients display varied severities of the disease and a diverse range of symptoms, but relatively invariant features include kidney failure, T-cell immunodeficiency, and skeletal dysplasia (Spranger et al. 1991; Boerkoel et al. 2000). In some cases, microcephaly also results (Deguchi et al. 2008), a phenotype that is often associated with defects in the DNA damage response at the organismal level $\left(\mathrm{O}^{\prime}\right.$ Driscoll and Jeggo 2008). Together, these intriguing phenotypes as well as the unique biochemical activity of HARP make it fascinating for further study.

\section{Insights into HARP function in the cell}

In this issue of Genes \& Development, four studies (Bansbach et al. 2009; Ciccia et al. 2009; Yuan et al. 2009; Yusufzai et al. 2009) shed light on HARP function in the cell, placing it physically and functionally at the replication fork during conditions of replicative stress and DNA damage. A major finding in all four studies is that HARP interacts with the RPA complex, and three of the studies indicate that this occurs via a direct interaction with RPA2. Indeed, sequence analysis of the conserved N terminus of HARP reveals notable homology with an RPA2-binding motif described previously in the replication checkpoint protein Tipin and DNA repair factors Ung2, XPA, and Rad52 (Mer et al. 2000; Unsal-Kacmaz et al. 2007). Importantly, this motif is necessary for the interaction of HARP with RPA2. Although not observed by Ciccia et al. (2009), work by Yuan et al. (2009) also suggests there is a direct interaction of HARP with RPA1 through the same region in HARP, indicating that HARP may interact with RPA at multiple sites. The interaction with RPA is constitutive and appears to be unaltered by
DNA damage (Ciccia et al. 2009; Yuan et al. 2009). In addition, mass spectrometry analysis indicates that the bulk of HARP is bound to RPA, suggesting that the interaction may be critical for HARP function (Ciccia et al. 2009).

Because the RPA complex plays a key role during DNA repair and DNA replication, all four groups sought to determine whether HARP localizes to sites of DNA damage and replication stress. The phosphorylated form of the histone variant $\mathrm{H} 2 \mathrm{AX}$ (termed $\gamma-\mathrm{H} 2 \mathrm{AX}$ ) is found at sites of damaged chromatin (Stucki et al. 2005), and together the groups find that HARP colocalizes with foci containing RPA and $\gamma$-H2AX when cells are treated with different DNA-damaging agents and replication inhibitors. Consistent with the idea that this localization is dependent on RPA, knockdown of RPA2 prevents the localization of HARP to sites of DNA damage (Ciccia et al. 2009). Furthermore, all groups show that mutants of HARP lacking the conserved RPA2-binding domain or bearing point mutations that prevent the interaction with RPA did not localize to sites of damage, whereas the RPAbinding domain alone could form nuclear foci. Taken together, these observations indicate that the conserved RPA interaction motif found within HARP is both necessary and sufficient for its localization to sites of DNA damage. These findings also raise the question of whether the RPA-HARP interaction is needed for the ability of HARP to catalyze the ATP-dependent reannealing of ssDNA bubbles coated with RPA in vitro. Interestingly, Bansbach et al. (2009) and Yusufzai et al. (2009) found that HARP protein lacking the conserved RPA-binding domain showed no defects in its annealing activity in vitro, nor was the ability of fork DNA to stimulate the ATPase activity compromised. These results indicate that the interaction of HARP with RPA may serve only to concentrate HARP activity at stalled or collapsed forks.

Interestingly, the localization of HARP to DNA damage appears to be cell cycle-specific. Ciccia et al. (2009) show that HARP recruitment to sites of laser-induced DNA damage occurs in $\sim 60 \%$ of cells, indicating that HARP, like RPA, may only be recruited to sites of damage in $S$ and $G_{2}$ phases of the cell cycle. Consistent with this idea, Bansbach et al. (2009) also show that HARP foci form following ionizing radiation (IR) only in S/G2-phase cells (stained with cyclin A). Both observations are consistent with the idea that HARP recognizes ssDNA generated during resection, a process that is restricted to $S$ phase and $G_{2}$ (Sartori et al. 2007). Indeed, localization of HARP to laser-induced sites of DNA damage required the DNA resection factor CtIP (Ciccia et al. 2009). Further indicating a possible cell cycle-specific role for HARP, Yuan et al. (2009), Ciccia et al. (2009), and Bansbach et al. (2009) assayed the ability of cells depleted of HARP to tolerate replicative stress. They found that HARP appears to be required for resistance to camptothecin (CPT), mitomycin $\mathrm{C}$, aphidicolin, and hydroxyurea $(\mathrm{HU})$, all agents that damage or stress replication forks. Ciccia et al. (2009) also report sensitivity to IR, although this was not observed by Yuan et al. (2009). 
The sensitivity of HARP-depleted cells to fork stalling agents, the S-phase-specific localization of HARP to sites of DNA damage, and the stimulation of HARP by fork DNA structures raise the possibility that HARP acts during $S$ phase or under conditions of replicative stress to stabilize or restart stalled forks or to repair collapsed forks. Such a defect might be expected to lead to problems in cell cycle progression and damage accumulation. Indeed, analysis of the cell cycle in HARP-depleted cells by Yuan et al. (2009) and Bansbach et al. (2009) revealed an accumulation in $\mathrm{G}_{2}-\mathrm{M}$. Furthermore, Ciccia et al. (2009) observed a defect in the progression of S-phase cells into mitosis, and this effect was exacerbated by treatment with IR and CPT. Yuan et al. (2009) and Banbach et al. (2009) also report that HARP-depleted cells accumulate RPA and $\gamma$-H2AX foci, and Yuan et al. (2009) observe activation of ATM and Chk2. Although indirect, these results suggest increased spontaneously arising DNA DSBs in the absence of HARP. Functional complementation of cell lines depleted of HARP indicate that both the RPA-binding domain of HARP as well as its helicase activities are needed to prevent these defects (Bansbach et al. 2009; Yuan et al. 2009). Together, these data suggest there is a requirement for HARP's activity in responding to and/or repairing DNA damage arising during $S$ phase, and that in its absence the breaks accumulate, slowing cell cycle progression through checkpoint activation.

To more directly investigate whether HARP may be required for the restart of stalled replication forks, Ciccia et al. (2009) also assayed the ability of HARP-deficient cells to restart replication after treatment with the replication inhibitor thymidine. They found that HARPdepleted cells exhibit a delay in BrdU incorporation following release. To distinguish between the possibility that this delay was due to a problem in fork restart or stabilization versus a problem with new origin firing, Ciccia et al. (2009) then analyzed fork progression during aphidicolin treatment on single DNA fibers (a technique that allows the examination of events at individual replication forks). Their analysis showed that HARPdepleted cells have a significant defect in the ability to restart existing replication forks after treatment with aphidicolin, whereas no defect in new origin firing was observed. Because DSBs appear to form in HARP-depleted cells, it seems likely that there is an increased number of collapsed forks in these cells. Taken together, these observations strongly implicate HARP in the fork restart process. Such a defect could result from an increased rate of fork collapse due to a role for HARP in fork stabilization, or from a failure of HARP to facilitate the repair of collapsed forks by homologous recombination.

\section{HARP at the fork}

So, how do these new results relate to the recently described ability of HARP to act as an annealing helicase? In order to maintain the stability of a stalled replication fork, HARP may prevent certain events at the fork to keep its integrity intact and allow eventual resumption of replication without the need for homologous recombination (Fig.
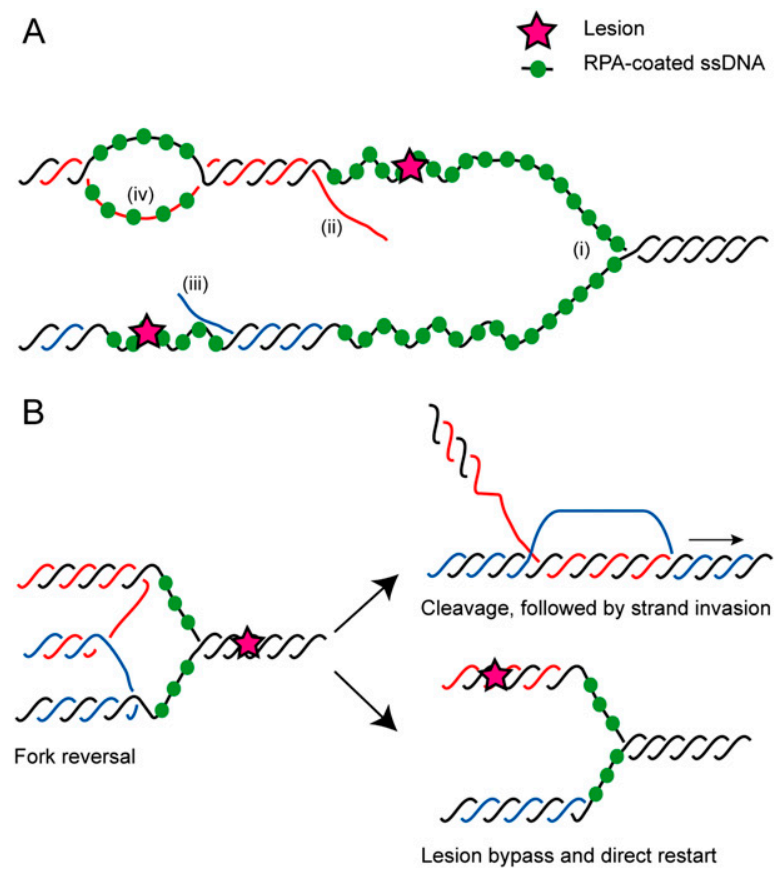

Figure 1. Potential targets of HARP activity at the replication fork. (A) HARP may act to prevent certain events at a stalled fork, stabilizing this structure. For example, it may limit helicasemediated DNA unwinding at stalled forks to prevent extensive accumulation of ssDNA that may disrupt a stable fork structure (i). Precocious dissociation of the DNA polymerases at unstable forks could also result in unwinding of the nascent strand on the leading (ii) and lagging (iii) strand, and HARP may suppress the formation of these structures. Such unwinding could lead to flap formation and unwanted fork regression or aberrant homologous recombination. HARP could also prevent bubble formation behind the fork at genomic regions that are not yet packaged into chromatin and are inherently unstable, such as A-T-rich regions (iv). (B) Alternatively, after fork stalling, HARP may promote fork regression (left) or certain fork configurations required for lesion bypass (bottom right), but it could also act during a recombination-mediated fork restart event such as break-induced replication (top right).

1A). There are multiple DNA structures on which an annealing helicase could act after the replication fork encounters DNA damage, one of which is the ssDNA, which accumulates at stalled replication forks when the activities of the helicase and stalled polymerases are uncoupled. In effect, HARP would counter helicase activity by reversing excessive unwinding (Fig. 1A, i). It is unclear, however, if it would be necessary for the MCM helicase to dissociate or "back up" for reannealing of the ssDNA strands to occur. Such dissociation could be problematic for replication restart because the cell employs a number of mechanisms to prevent MCM loading in S phase (Blow and Dutta 2005). The premature dissociation of polymerases at a stalled fork could result in a second class of possible substrates for HARP. In this scenario, 3 '-DNA flaps could form on either the leading or lagging strand due to dissociation of DNA polymerases (Fig. 1A, ii,iii), and in the presence of $5^{\prime}$-DNA flaps, which can form on Okazaki fragments, fork reversal could occur, 
allowing excess cleavage and recombination to occur. A third possible type of substrate could exist behind the fork in the form of ssDNA bubbles, the substrate initially used to demonstrate the annealing activity of HARP. Although nucleosome formation behind the fork is coupled to fork progression (Groth et al. 2007), bubble structures could form, especially in A-T-rich regions or loci prone to secondary structure formation (Fig. 1A, iv), potentially allowing inappropriate processing of the DNA.

HARP may also act to promote certain events at the fork in order to facilitate restart of a stalled fork or repair of a broken fork (Fig. 1B). Although HARP does not exhibit helicase activity on partial duplex substrates in vitro (Yusufzai and Kadonaga 2008), it is formally possible that its ATP-dependent DNA translocation coupled to its annealing helicase activity could be used to anneal nascent strands, allowing fork regression in vivo, in a similar manner to that of Rad5, a related Snf2 family member (Fig. 1B, left; Atkinson and McGlynn 2009). Data from studies in $S$. cerevisiae suggest that formation of regressed forks might be a rare and pathological event in eukaryotic cells (Sogo et al. 2002). Nevertheless, these structures have been implicated in replication restart models involving lesion bypass (Heller and Marians 2006). In fact, HARP could also help reset the nascent strands after fork regression (Fig. 1B, bottom right). Finally, it has been proposed that cleavage of regressed forks can generate the substrate required for recombination-dependent fork restart (such as break-induced replication (Fig. 1B, top right; Heller and Marians 2006), and Ciccia et al. (2009) suggest that HARP may play a role during this replication-specific recombination event. Although their data demonstrate that HARP is not required for gene conversion, Ciccia et al. (2009) speculate that the function may be linked specifically to lagging strand synthesis, which is not required for gene conversion.

Clearly, further work will be needed to determine the substrate on which HARP acts in cells. On this point, it is interesting that Bansbach et al. (2009) found that both HARP loss and overexpression led to H2AX phosphorylation. In the case of overexpression as opposed to its loss, nuclear foci of $\gamma-\mathrm{H} 2 \mathrm{AX}$ were not observed, and instead a pan-nuclear staining was observed-again, in S-phase cells. Furthermore, the DNA damage response did not appear to be activated under conditions of HARP overexpression, while it was upon HARP depletion. Although the majority of HARP is bound to RPA, only a small fraction of RPA is bound to HARP. Thus, overexpression could act indirectly by altering the distribution of RPA complexes found at normal forks. However, because the $\gamma$-H2AX signal is dependent on the ATPase as well as RPA-binding activities of HARP, these observations suggest that HARP is instead acting on a common structure present at all forks to which it normally does not have sufficient access.

\section{Future questions}

Together, the four new studies in this issue of Genes \& Development (Bansbach et al. 2009; Ciccia et al. 2009;
Yuan et al. 2009; Yusufzai et al. 2009) provide important insights into the function of a protein centrally located at the replication fork. These studies, however, raise some fascinating questions beyond the critical question of what substrate HARP acts on in cells. The RPA-binding domain of HARP is highly similar to that of the replication response protein Tipin, and the binding region on RPA is shared among multiple repair proteins, including Rad52 and XPA (Mer et al. 2000). Exactly how RPA coordinates the activities of all these proteins temporally during the DNA damage response and with regard to its multiple roles in the cell will be interesting to elucidate. What determines which RPA complexes move to the fork or lesion in a given situation? Is this a stochastic process, or is additional regulation required? One possibility is that the phosphorylation of RPA, known to occur during $S$ phase and following DNA damage, may play a role in this selection (Zou 2007). Phosphorylation of RPA's binding partners could be involved. Indeed, Bansbach et al. (2009) show that HARP is phosphorylated in a phosphoinositide-3-kinase-related protein kinasedependent manner, and it will certainly be interesting to determine the function of this modification, particularly with regard to its regulation of HARP's ATPase and helicase activities as well as its localization.

Because the RPA complex plays a crucial role in activation of the replication checkpoint, it may also be interesting to determine how HARP may affect the activity of ATR. If HARP acts to prevent ssDNA formation (as expected from its in vitro activity), one might imagine that HARP may be important for limiting checkpoint activity at the fork. Two groups (Bansbach et al. 2009; Yuan et al. 2009) did find that loss of HARP leads to the persistence of RPA foci and its chromatin association, suggesting that it does prevent ssDNA formation in cells.

Finally, insight into the role of HARP may come from defining the origin of the events that lead to DSB accumulation in HARP-depleted cells. A defect in fork stabilization leading to increased fork collapse is one possible mechanism. Another possibility is that the inability of cells to repair naturally occurring endogenous DSBs during replication results in their accumulation. Finally, these breaks could arise as a result of a programmed fork restart process. Mus81 has been shown recently to be involved in the formation of DSBs after fork stalling (Hanada et al. 2007), and it is also needed for fork recovery (Ciccia et al. 2008). Thus, the breaks found in the absence of HARP could be a result of Mus81 activity. This would suggest that accumulation of ssDNA at the fork in HARP-depleted cells may result in the stimulation of nucleases, like Mus81, and subsequent recombination.

Given the broad range of defects that HARP-depleted cells exhibit, defining exactly how HARP acts at stalled or broken replication forks will likely reveal exciting details about the complex nature of the cellular response to replication stress as well as a further understanding of the circumstances leading to the development of SIOD. 


\section{Acknowledgments}

This work was supported by grants to K.A.C. from the National Institutes of Health (ES016486 and ES016867), and by a grant to R.D. from the Jane Coffin Childs Memorial Fund for Medical Research. K.A.C. is a Leukemia and Lymphoma Scholar and a Ellison Medical Foundation Senior Scholar in Aging, and R.D. is a fellow of the Jane Coffin Childs Memorial Fund for Medical Research.

\section{Note added in proof}

Recent work from L. Postow, E.M. Woo, B.T. Chait, and H. Funabiki (in prep.) on Xenopus and human HARP/SMARCAL1 is consistent with the findings reported here.

\section{References}

Admire A, Shanks L, Danzl N, Wang M, Weier U, Stevens W, Hunt E, Weinert T. 2006. Cycles of chromosome instability are associated with a fragile site and are increased by defects in DNA replication and checkpoint controls in yeast. Genes \& Dev 20: 159-173.

Atkinson J, McGlynn P. 2009. Replication fork reversal and the maintenance of genome stability. Nucleic Acids Res 37: 3475-3492.

Bansbach CE, Bétous R, Lovejoy CA, Glick GG, Cortez D. 2009. The annealing helicase SMARCAL1 maintains genome integrity at stalled replication forks. Genes \& Dev (this issue). doi: $10.1101 / \mathrm{gad} .1839909$.

Barbour L, Xiao W. 2003. Regulation of alternative replication bypass pathways at stalled replication forks and its effects on genome stability: A yeast model. Mutat Res 532: 137-155.

Blastyak A, Pinter L, Unk I, Prakash L, Prakash S, Haracska L. 2007. Yeast Rad5 protein required for postreplication repair has a DNA helicase activity specific for replication fork regression. Mol Cell 28: 167-175.

Blow JJ, Dutta A. 2005. Preventing re-replication of chromosomal DNA. Nat Rev Mol Cell Biol 6: 476-486.

Boerkoel CF, O'Neill S, Andre JL, Benke PJ, Bogdanovic R, Bulla M, Burguet A, Cockfield S, Cordeiro I, Ehrich JH, et al. 2000. Manifestations and treatment of Schimke immuno-osseous dysplasia: 14 new cases and a review of the literature. Eur J Pediatr 159: 1-7.

Branzei D, Foiani M. 2005. The DNA damage response during DNA replication. Curr Opin Cell Biol 17: 568-575.

Branzei D, Foiani M. 2007. Interplay of replication checkpoints and repair proteins at stalled replication forks. DNA Repair (Amst) 6: 994-1003.

Branzei D, Foiani M. 2009. The checkpoint response to replication stress. DNA Repair (Amst) 8: 1038-1046.

Chang DJ, Cimprich KA. 2009. DNA damage tolerance: When it's OK to make mistakes. Nat Chem Biol 5: 82-90.

Ciccia A, McDonald N, West SC. 2008. Structural and functional relationships of the XPF/MUS81 family of proteins. Annu Rev Biochem 77: 259-287.

Ciccia A, Bredemeyer AL, Sowa ME, Terret M-E, Jallepalli PV, Harper JW, Elledge SJ. 2009. The SIOD disorder protein SMARCAL1 is an RPA-interacting protein involved in replication fork restart. Genes \& Dev (this issue). doi: 10.1101/ gad.1832309.

Cimprich KA, Cortez D. 2008. ATR: An essential regulator of genome integrity. Nat Rev Mol Cell Biol 9: 616-627.

Cobb JA, Bjergbaek L, Shimada K, Frei C, Gasser SM. 2003. DNA polymerase stabilization at stalled replication forks requires Mec1 and the RecQ helicase Sgs1. EMBO I 22: 4325-4336.
Cortez D, Glick G, Elledge SJ. 2004. Minichromosome maintenance proteins are direct targets of the ATM and ATR checkpoint kinases. Proc Natl Acad Sci 101: 10078-10083.

Deguchi K, Clewing JM, Elizondo LI, Hirano R, Huang C, Choi K, Sloan EA, Lucke T, Marwedel KM, Powell RD Jr, et al. 2008. Neurologic phenotype of Schimke immuno-osseous dysplasia and neurodevelopmental expression of SMARCAL1. I Neuropathol Exp Neurol 67: 565-577.

Delacroix S, Wagner JM, Kobayashi M, Yamamoto K, Karnitz LM. 2007. The Rad9-Hus1-Rad1 (9-1-1) clamp activates checkpoint signaling via TopBP1. Genes \& Dev 21: 14721477.

Driscoll R, Hudson A, Jackson SP. 2007. Yeast Rtt109 promotes genome stability by acetylating histone H3 on lysine 56. Science 315: 649-652.

Durr H, Flaus A, Owen-Hughes T, Hopfner KP. 2006. Snf2 family ATPases and DExx box helicases: Differences and unifying concepts from high-resolution crystal structures. Nucleic Acids Res 34: 4160-4167.

Ellison V, Stillman B. 2003. Biochemical characterization of DNA damage checkpoint complexes: Clamp loader and clamp complexes with specificity for $5^{\prime}$ recessed DNA. PLoS Biol 1: E33. doi: 10.1371/journal.pbio.0000033.

Flaus A, Martin DM, Barton GJ, Owen-Hughes T. 2006. Identification of multiple distinct Snf2 subfamilies with conserved structural motifs. Nucleic Acids Res 34: 2887-2905.

Groth A, Corpet A, Cook AJ, Roche D, Bartek J, Lukas J, Almouzni G. 2007. Regulation of replication fork progression through histone supply and demand. Science 318: 1928-1931.

Han I, Zhou H, Horazdovsky B, Zhang K, Xu RM, Zhang Z. 2007a. Rtt109 acetylates histone H3 lysine 56 and functions in DNA replication. Science 315: 653-655.

Han J, Zhou H, Li Z, Xu RM, Zhang Z. 2007b. Acetylation of lysine 56 of histone H3 catalyzed by RTT109 and regulated by ASF1 is required for replisome integrity. J Biol Chem 282: 28587-28596.

Hanada K, Budzowska M, Davies SL, van Drunen E, Onizawa H, Beverloo HB, Maas A, Essers J, Hickson ID, Kanaar R. 2007. The structure-specific endonuclease Mus81 contributes to replication restart by generating double-strand DNA breaks. Nat Struct Mol Biol 14: 1096-1104.

Heller RC, Marians KJ. 2006. Replisome assembly and the direct restart of stalled replication forks. Nat Rev Mol Cell Biol 7: 932-943.

Hickson ID. 2003. RecQ helicases: Caretakers of the genome. Nat Rev Cancer 3: 169-178.

Hockensmith JW, Wahl AF, Kowalski S, Bambara RA. 1986. Purification of a calf thymus DNA-dependent adenosinetriphosphatase that prefers a primer-template junction effector. Biochemistry 25: 7812-7821.

Kastan MB, Bartek J. 2004. Cell-cycle checkpoints and cancer. Nature 432: 316-323.

Katou Y, Kanoh Y, Bando M, Noguchi H, Tanaka H, Ashikari T, Sugimoto K, Shirahige K. 2003. S-phase checkpoint proteins Tof1 and Mrc1 form a stable replication-pausing complex. Nature 424: 1078-1083.

Kolodner RD, Putnam CD, Myung K. 2002. Maintenance of genome stability in Saccharomyces cerevisiae. Science 297: 552-557.

Kumagai A, Lee J, Yoo HY, Dunphy WG. 2006. TopBP1 activates the ATR-ATRIP complex. Cell 124: 943-955.

Lambert S, Carr AM. 2005. Checkpoint responses to replication fork barriers. Biochimie 87: 591-602.

Majka J, Burgers PM. 2004. The PCNA-RFC families of DNA clamps and clamp loaders. Prog Nucleic Acid Res Mol Biol 78: $227-260$. 
Majka J, Binz SK, Wold MS, Burgers PM. 2006. Replication protein A directs loading of the DNA damage checkpoint clamp to 5'-DNA junctions. I Biol Chem 281: 27855-27861.

Masumoto H, Hawke D, Kobayashi R, Verreault A. 2005. A role for cell-cycle-regulated histone $\mathrm{H} 3$ lysine 56 acetylation in the DNA damage response. Nature 436: 294-298.

McGlynn P, Lloyd RG. 2002. Replicating past lesions in DNA. Mol Cell 10: 700-701.

Mer G, Bochkarev A, Gupta R, Bochkareva E, Frappier L, Ingles CJ, Edwards AM, Chazin WJ. 2000. Structural basis for the recognition of DNA repair proteins UNG2, XPA, and RAD52 by replication factor RPA. Cell 103: 449-456.

Mordes DA, Glick GG, Zhao R, Cortez D. 2008. TopBP1 activates ATR through ATRIP and a PIKK regulatory domain. Genes \& Dev 22: 1478-1489.

Muthuswami R, Truman PA, Mesner LD, Hockensmith JW. 2000. A eukaryotic SWI2/SNF2 domain, an exquisite detector of double-stranded to single-stranded DNA transition elements. I Biol Chem 275: 7648-7655.

O'Driscoll M, Jeggo PA. 2008. The role of the DNA damage response pathways in brain development and microcephaly: Insight from human disorders. DNA Repair (Amst) 7: 1039-1050.

Olson E, Nievera CJ, Klimovich V, Fanning E, Wu X. 2006. RPA2 is a direct downstream target for ATR to regulate the S-phase checkpoint. J Biol Chem 281: 39517-39533.

Papamichos-Chronakis M, Peterson CL. 2008. The Ino80 chromatin-remodeling enzyme regulates replisome function and stability. Nat Struct Mol Biol 15: 338-345.

Paques F, Haber JE. 1999. Multiple pathways of recombination induced by double-strand breaks in Saccharomyces cerevisiae. Microbiol Mol Biol Rev 63: 349-404.

Pacek M, Walter JC. 2004. A requirement for MCM7 and Cdc45 in chromosome unwinding during eukaryotic DNA replication. EMBO J 23: 3667-3676.

Paulsen RD, Cimprich KA. 2007. The ATR pathway: Finetuning the fork. DNA Repair (Amst) 6: 953-966.

Sartori AA, Lukas C, Coates J, Mistrik M, Fu S, Bartek J, Baer R, Lukas J, Jackson SP. 2007. Human CtIP promotes DNA end resection. Nature 450: 509-514.

Shimada K, Oma Y, Schleker T, Kugou K, Ohta K, Harata M, Gasser SM. 2008. Ino80 chromatin remodeling complex promotes recovery of stalled replication forks. Curr Biol 18: 566-575.

Smirnova M, Klein HL. 2003. Role of the error-free damage bypass postreplication repair pathway in the maintenance of genomic stability. Mutat Res 532: 117-135.

Smith KD, Fu MA, Brown E. 2009. Tim/Tipin dysfunction creates an indispensible reliance on the ATR-Chk1 pathway for continued DNA synthesis. I Cell Biol (in press).

Sogo JM, Lopes M, Foiani M. 2002. Fork reversal and ssDNA accumulation at stalled replication forks owing to checkpoint defects. Science 297: 599-602.

Spranger J, Hinkel GK, Stoss H, Thoenes W, Wargowski D, Zepp F. 1991. Schimke immuno-osseous dysplasia: A newly recognized multisystem disease. I Pediatr 119: 64-72.

Stucki M, Clapperton JA, Mohammad D, Yaffe MB, Smerdon SJ, Jackson SP. 2005. MDC1 directly binds phosphorylated histone H2AX to regulate cellular responses to DNA doublestrand breaks. Cell 123: 1213-1226.

Tittel-Elmer M, Alabert C, Pasero P, Cobb JA. 2009. The MRX complex stabilizes the replisome independently of the $S$ phase checkpoint during replication stress. EMBO $J$ 28: 1142-1156.

Tourriere H, Pasero P. 2007. Maintenance of fork integrity at damaged DNA and natural pause sites. DNA Repair (Amst) 6: 900-913.
Unsal-Kacmaz K, Chastain PD, Qu PP, Minoo P, Cordeiro-Stone M, Sancar A, Kaufmann WK. 2007. The human Tim/Tipin complex coordinates an intra-S checkpoint response to UV that slows replication fork displacement. Mol Cell Biol 27: 3131-3142.

Yuan J, Ghosal G, Chen J. 2009. The annealing helicase HARP protects stalled replication forks. Genes \& Dev (this issue). doi: 10.1101/gad.1836409.

Yusufzai T, Kadonaga JT. 2008. HARP is an ATP-driven annealing helicase. Science 322: 748-750.

Yusufzai T, Kong X, Yokomori K, Kadonaga JT. 2009. The annealing helicase HARP is recruited to DNA repair sites via an interaction with RPA. Genes \& Dev (this issue). doi: $10.1101 /$ gad.1831509.

Zou L. 2007. Single- and double-stranded DNA: Building a trigger of ATR-mediated DNA damage response. Genes \& Dev 21: 879-885.

Zou L, Elledge SJ. 2003. Sensing DNA damage through ATRIP recognition of RPA-ssDNA complexes. Science 300: 15421548.

Zou Y, Liu Y, Wu X, Shell SM. 2006. Functions of human replication protein A (RPA): From DNA replication to DNA damage and stress responses. J Cell Physiol 208: 267-273. 


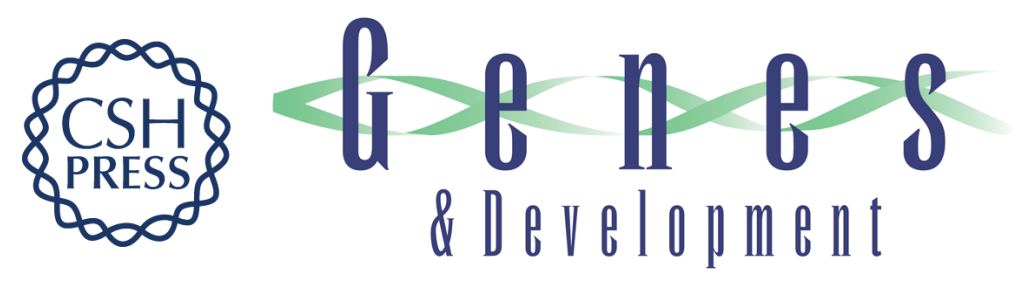

\section{HARPing on about the DNA damage response during replication}

Robert Driscoll and Karlene A. Cimprich

Genes Dev. 2009, 23:

Access the most recent version at doi:10.1101/gad.1860609

Related Content The annealing helicase SMARCAL1 maintains genome integrity at stalled replication forks

Carol E. Bansbach, Rémy Bétous, Courtney A. Lovejoy, et al.

Genes Dev. October, 2009 23: 2405-2414 The SIOD disorder protein SMARCAL1 is

an RPA-interacting protein involved in replication fork restart

Alberto Ciccia, Andrea L. Bredemeyer, Mathew E. Sowa, et al.

Genes Dev. October , 2009 23: 2415-2425 The annealing helicase HARP protects

stalled replication forks

Jingsong Yuan, Gargi Ghosal and Junjie Chen

Genes Dev. October , 2009 23: 2394-2399 The annealing helicase HARP is recruited

to DNA repair sites via an interaction with RPA

Timur Yusufzai, Xiangduo Kong, Kyoko Yokomori, et al.

Genes Dev. October, 2009 23: 2400-2404

References This article cites 62 articles, 24 of which can be accessed free at:

http://genesdev.cshlp.org/content/23/20/2359.full.html\#ref-list-1

Articles cited in:

http://genesdev.cshlp.org/content/23/20/2359.full.html\#related-urls

\section{License}

Email Alerting

Receive free email alerts when new articles cite this article - sign up in the box at the top Service

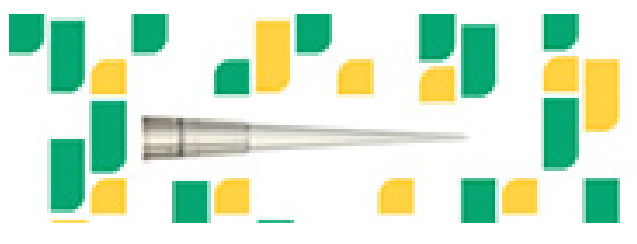

Focused on your science. 